\title{
Large-scale survey for novel genotypes of Plasmodium falciparum chloroquine-resistance gene pfcrt
}

\begin{abstract}
Nobuyuki Takahashi ${ }^{1}$, Kazuyuki Tanabe ${ }^{2}$, Takahiro Tsukahara', Mawuli Dzodzomenyo ${ }^{3}$, Lek Dysoley ${ }^{4}$, Boualam Khamlome ${ }^{5}$, Jetsumon Sattabongkot ${ }^{6}$, Masatoshi Nakamura ${ }^{7}$, Miki Sakurai $^{1}$, Jun Kobayashi ${ }^{8}$, Akira Kaneko 9,10,11, Hiroyoshi Endo ${ }^{1}$, Francis Hombhanje ${ }^{12}$, Takafumi Tsuboi ${ }^{13}$ and Toshihiro Mita ${ }^{1 *}$
\end{abstract}

\begin{abstract}
Background: In Plasmodium falciparum, resistance to chloroquine (CQ) is conferred by a $\mathrm{K}$ to T mutation at amino acid position 76 (K76T) in the P. falciparum CQ transporter (PfCRT). To date, at least 15 pfcrt genotypes, which are represented by combinations of five amino acids at positions 72-76, have been described in field isolates from various endemic regions. To identify novel mutant pfcrt genotypes and to reveal the genetic relatedness of pfcrt genotypes, a large-scale survey over a wide geographic area was performed.

Methods: Sequences for exon 2 in pfcrt, including known polymorphic sites at amino acid positions 72, 74, 75 and 76, were obtained from 256 P. falciparum isolates collected from eight endemic countries in Asia (Bangladesh, Cambodia, Lao P.D.R., the Philippines and Thailand), Melanesia (Papua New Guinea and Vanuatu) and Africa (Ghana). A haplotype network was constructed based on six microsatellite markers located $-29 \mathrm{~kb}$ to $24 \mathrm{~kb}$ from pfcrt in order to examine the genetic relatedness among mutant pfcrt genotypes.

Results: In addition to wild type (CVMNK at positions 72-76), four mutant pfcrt were identified; CVIET, CVIDT, SVMNI and CVMNI (mutated amino acids underlined). Haplotype network revealed that there were only three mutant pfcrt lineages, originating in Indochina, Philippines and Melanesia. Importantly, the Indochina lineage contained two mutant pfcrt genotypes, CVIET $(n=95)$ and CVIDT $(n=14)$, indicating that CVIDT shares a common origin with CVIET. Similarly, one major haplotype in the Melanesian lineage contained two pfcrt genotypes; SVMNT $(n=71)$ and CVMNT $(n=3)$. In Africa, all mutant pfcrt genotypes were the CVIET of the Indochina lineage, probably resulting from the intercontinental migration of CQ resistance from Southeast Asia.

Conclusions: The number of CQ-mutant lineages observed in this study was identical to that found in previous studies. This supports the hypothesis that the emergence of novel CQ resistance is rare. However, in the mutant pfrt genotypes, amino acid changes at positions 72, 74 and 75 appear to have recently been generated at least several times, producing distinct pfcrt mutant genotypes. The occurrence of new mutations flanking K76T may yield stronger resistance to $C Q$ and/or a higher fitness than the original pfcrt mutant.
\end{abstract}

Keywords: Plasmodium falciparum, Chloroquine resistance, pfcrt, Microsatellite, Haplotype network, Evolution

\footnotetext{
* Correspondence: hiro-tm@research.twmu.ac.jp

'Department of International Affairs and Tropical Medicine, Tokyo Women's Medical University School of Medicine, 8-1 Kawada-cho, Shinjuku-ku, Tokyo 162-8666, Japan

Full list of author information is available at the end of the article
} 


\section{Background}

The spread of drug-resistant Plasmodium falciparum, the most virulent malaria parasite, represents a serious concern for the treatment and control of falciparum malaria. It is generally believed that the emergence of drug-resistant $P$. falciparum is rare and geographically restricted [1-4]. Clinical resistance to chloroquine (CQ) was first identified simultaneously in two different geographic regions in the late 1950s; Southeast Asia (Thailand-Cambodia border) [5] and South America [6]. CQ resistance then expanded to neighbouring countries in the 1960s, and nearly all Southeast Asian countries by the mid-1970s [4]. In Melanesia, resistance to CQ was reported in the early 1960s in Indonesian West Papua, shortly after mass administration of CQ in medicated table salt [7]. Subsequently, it spread to Papua New Guinea [8] and the Solomon Islands [4] in 1976 and 1980, respectively. In Africa, CQ resistance was first reported in the late 1970s in Tanzania $[9,10]$, and it was found to be have been introduced from Southeast Asia [11].

Since the discovery of $P$. falciparum chloroquine transporter (PfCRT) as a primary target of CQ resistance [12], reports on the geographic origins and spread of CQ-resistant P. falciparum have accumulated [1]. PfCRT is localized to the parasite food vacuole and is known to have $>10$ polymorphic amino acid sites [12]. Among these, an amino acid change from Lys (K) to Thr $(\mathrm{T})$ at position $76(\mathrm{~K} 76 \mathrm{~T})$ plays a decisive role in conferring resistance to CQ [12]; the mutation greatly reduces the accumulation of CQ in the parasite food vacuole by accelerating efflux of CQ [13]. Microsatellite (MS) analysis flanking the PfCRT gene, pfcrt, has revealed that the geographic origin of $C Q$ resistance is quite limited, with only four CQ resistant lineages initially identified: one in Indochina/Africa, one in Melanesia, and two in South America (Brazil/Peru and Ecuador/Colombia) [1]. Subsequently, one distinct CQresistant lineage was discovered in isolates originating in the Philippines [14].

These CQ resistant lineages harbour one of four mutant pfcrt genotypes at positions 72-76 (CVIET, $\underline{\text { SVMNT}}$, CVMNT and CVMET; mutations underlined), with the SVMNT genotype being found in Brazil/Peru and Melanesia lineages $[1,14]$. In addition, at least 10 mutant $p f c r t$ genotypes have recently been identified in field isolates from various endemic regions; SVMIT (Guyana [15]), SVMET (Colombia [16]), SVIET (Indonesian Papua [17]), SVMDT (Philippines [18]), CVMET (Colombia [16]), CVMNN (Indonesia [19]), CVT $\underline{\mathrm{NT}}$ (Cambodia [20]), CVIDT (Madagascar [21], India [22], Cambodia [20]), CVMDT (Philippines [18]) and RVMNT (Guyana [15]). Meanwhile, recovery of CQ sensitivity was reported after the use of CQ was abandoned in Malawi [23-25]. Similar recovery has also been reported on Hainan Island, China [26]. This recovery was thought to be due to the re-introduction of susceptible parasites harbouring a CQ-sensitive pfcrt [27,28], but a back mutation in pfcrt at position 76 from the resistant-type amino acid ( $\mathrm{T}$ ) to the sensitive-type amino acid (K) may also be potentially involved in the recovery of CQ sensitivity in some endemic areas, although this has yet to be confirmed.

This possibility can be inferred from the previous finding of a laboratory-maintained CQ-sensitive P. falciparum clone (106/1) originating in Sudan that apparently underwent a back mutation from $\mathrm{T}$ to $\mathrm{K}$ at position 76 [12]. It is thus likely that novel pfcrt genotypes may be identified through surveys of large numbers of $P$. falciparum isolates from diverse geographic areas. In support of this, recent large-scale surveys of genotypes of the dihydrofolate reductase gene $d h f r$, a target of pyrimethamine, and of the dihydropteroate gene dhps, a target of sulphadoxine, have identified several novel genotypes from wide geographic areas [24,25], and have provided insights into the evolutionary history of drug resistance in P. falciparum.

In this study, genotyping of $p f c r t$ and haplotyping of MS markers flanking pfcrt were performed in P. falciparum isolates collected from Asia (Bangladesh, Cambodia, Lao P.D.R., Philippines and Thailand), Melanesia (Papua New Guinea and Vanuatu), and Africa (Ghana). Results showed that there were only three CQ-resistant lineages, all of which were identified previously [1,14]. No evidence of back mutation was observed at position 76. Importantly, however, among pfcrt genotypes having the K76T mutation, amino acid changes other than K76T appear to have been recently generated on at least several occasions, producing novel $p f c r t$ mutant genotypes.

\section{Methods}

\section{Study area and samples}

Blood samples were obtained from $P$. falciparuminfected individuals living in eight malaria endemic countries as follows.

(1) Thailand: isolates were obtained during a longitudinal study on malaria transmission at a village in Kanchanaburi Province located at the western border of Thailand. Pre-treatment venous blood from a falciparum malaria-positive villager was obtained for this study between 2000 and 2003.

(2) Lao P.D.R.: isolates were specifically obtained for this study. Finger-pricked blood samples were taken in Napong Village, Boulapha District, Khammouane Province, in March 1999.

(3) Cambodia: isolates were obtained from finger-prick blood samples taken in Chumkiri District, located in the 
southeastern coastal Province of Kampot, in December 2004 [29].

(4) Bangladesh: isolates were obtained from fingerprick blood samples taken in Bandarban district hospital from October to December 2007 and six malaria endemic villages; Sultanpur, Chemidalupara, Kyaching ghata natun para, Saingya daneshpara, Faruqpara and Empupara, in Bandarban District, in March 2008 [30].

(5) Philippines: isolates were obtained from pre-treatment venous blood samples taken on Palawan Island, Palawan Province, in May and October 1997 [31].

(6) Papua New Guinea: isolates were obtained from finger-prick blood samples taken during in vitro studies at Wewak General Hospital located in the Wewak District, East Sepik Province, in 2002 and 2003 [32].

(7) Vanuatu: isolates were obtained from finger-prick blood samples taken on Gaua Island, in February 1997, Pentecost Island, in February 1998 [33].

(8) Ghana: isolates were obtained from finger-prick blood samples taken from three villages, Okyereko, Mpota and Apam, near Winneba, a western coastal region, in November 2004 [4].

\section{Ethical considerations}

Before enrolment, written informed consent was obtained from all study subjects. In the case of children, consent was obtained from a parent or legal guardian. This study was approved by (1) The Institutional Ethics Committee of the Thai Ministry of Public Health, the Human Subjects Research Review Board of the United States Army, (2) The Center of Malariology, Parasitology and Entomology (CMPE), Lao P.D.R., the Research Committee of the Ministry of Public Health (MoPH), Lao P.D.R., (3) The National Center for Parasitology, Entomology and Malaria Control (CNM), Cambodia, (4) The Bangladesh Medical Research Council and the local regulatory body of health in Bandarban, Bangladesh, (5) The Palawan Provincial Health Office, Philippines, (6) The National Department of Health Medical Research Advisory Committee of Papua New Guinea, (7) The Vanuatu Department of Health, Vanuatu, and (8) The Ministry of Health/Ghana Health Service, Ghana.

\section{DNA extraction}

Finger-prick blood was spotted onto chromatography filter paper ET31CHR (Whatman, Maidstone, UK) in Lao P.D.R., Cambodia, Bangladesh, Papua New Guinea, Vanuatu and Ghana. In Thailand and the Philippines, venous blood was transferred into heparin-containing test tubes. Parasite DNA was extracted using QIAamp DNA mini kits (Qiagen, Hilden, Germany) from a quarter of a dried blood spot or a corresponding amount of blood $(25 \mu \mathrm{l})$, in accordance with the manufacturer's instructions. The Lao P.D.R. and Bangladesh samples were extracted using QIAamp DNA mini kits with the QIAcube $^{\mathrm{TM}}$ (Qiagen) tissue protocol.

\section{pfcrt genotyping}

The $p f c r t$ gene was amplified by nested PCR using two sets of primers designed to amplify a region of exon 2 including known polymorphic sites at amino acid positions $72,74,75$ and 76 . PCR primers were designed after the sequence of 3D7 clone [GenBank: NC_004328]. Primer sequences and PCR conditions are shown in Additional file 1. Amplified product (aa 57-120) was purified using ExoSAP-IT (GE Healthcare UK Ltd., Buckinghamshire, UK) and were directly sequenced with a DYEnamic ET terminator kit in the MegaBACE 1000 DNA sequencer (GE Healthcare UK Ltd.). Data on pfcrt genotypes previously obtained from Papua New Guinea were included in the present analysis [32].

\section{Microsatellite haplotyping}

Variations in the number of TA repeats located at 0.59 $\mathrm{kb}, 10.389 \mathrm{~kb}, 23.576 \mathrm{~kb},-2.814 \mathrm{~kb}$ and $-29.268 \mathrm{~kb}$ in pfcrt and ATT repeats located at $10.389 \mathrm{~kb}$ in pfcrt were measured using PCR protocol previously described with some modifications (Additional file 2) [34]. Briefly, each MS marker was amplified by semi-nested PCR using fluorescent 5'-end labeled primers (Applied Biosystems., Foster city, CA, USA) in an ABI 2720 thermal cycler (Applied Biosystems). Amplified products were analysed using an ABI 377 DNA sequencer and GeneScan 3.1.2 software with GENESCAN ${ }^{\text {TM }} 400$ HD ROX size standard (Applied Biosystems), followed by size determination using a Genotyper 2.0 (Applied Biosystems). When two or more polymorphisms were detected, these isolates were considered to be mixed infections and excluded them from further analysis. As it has been well documented that wild-type parasites show extensive MS variations due to the lack of selective CQ sweeps [1], MS haplotypes were determined only for $p f c r t$ mutant isolates.

\section{Phylogenetic analysis}

In order to assess the genetic relationships among CQresistant $p f c r t$ genotypes, a median-joining haplotype network was constructed based on alleles at the six MS loci using the Network 4.6 software [35]. Median joining is a method for constructing genetic networks to identify the minimum spanning tree by favouring short connections [35].

\section{Results}

\section{pfcrt genotypes}

Among 263 P. falciparum isolates examined in this study, pfcrt genotypes were successfully determined in 256 samples. In addition to the wild-type CVMNK 
(10\%), four pfcrt mutant genotypes were identified; CVIET (48\%), CVIDT (6\%), SVMNT (33\%) and CVMNT (1\%). Mixed genotypes were observed in four samples (2\%) (Figure 1). In endemic regions of Indochina (Thai and Cambodia), South Asia (Bangladesh) and Africa (Ghana), CVIET was the predominant mutant genotype, which is consistent with previous studies $[1,11]$. One exception was Lao P.D.R., where the wild genotype and CVIDT were equally predominant, with CVIET showing a low frequency. In the Philippines, as previously observed [14], SVMNT was the dominant mutant genotype, and CVMNT was also found. In Melanesia, only SVMNT was found as a mutant genotype, as reported previously [36]. We did not observe any novel mutations at amino acid positions 57-120, other than those known at positions $72,74,75$ and 76 .

\section{Microsatellite haplotypes and network analysis}

Among 227 isolates harbouring pfcrt mutant genotypes, 196 samples were successfully determined for MS haplotypes. In total, 49 MS haplotypes were identified, including the three previously known major haplotypes (IC1, M1 and P1) and those haplotypes similar to IC1, M1 or
P1 (Figure 2). To assess the genetic relatedness of $p f c r t$ mutants, a haplotype network based on size variations at all MS markers was constructed. The haplotype network clearly showed three distinct clusters of $p f c r t$ mutants (Figure 3). The first lineage (Indochina lineage) consisted of the most prevalent haplotype IC1 $(\mathrm{n}=57)$, having a combination of alleles of 152-180-182-150-203191 at the MS loci of $-29.268 \mathrm{~kb},-10.833 \mathrm{~kb},-2.814 \mathrm{~kb}$, $0.59 \mathrm{~kb}, 10.389 \mathrm{~kb}$ and $23.576 \mathrm{~kb}$, respectively, and those haplotypes closely related to IC1. Nearly all isolates in this lineage harboured the CVIET genotype. However, several isolates (IC1, IC5, IC6, IC9 and IC10; $\mathrm{n}=14$ ) contained the CVIDT genotype. This indicates that this mutant genotype shares a common origin with CVIET, and that MS haplotype IC1 contained two mutant pfcrt genotypes. The second lineage (Melanesian lineage) contained the most prevalent haplotype M1 (n = 36), having an allele combination of 152-172-182-152206-187 and those haplotypes related to M1. All but M2 $(\mathrm{CVMN} \underline{\mathrm{T}}, \mathrm{n}=3)$ harboured the SVMNT genotype. This indicates that MS haplotype $\bar{M} 1$ also contained two mutant pfcrt genotypes. In the third lineage (Philippine lineage), all but one (P2) harboured an allele combination of 152-170-190-142-200-189 (P1). As previously

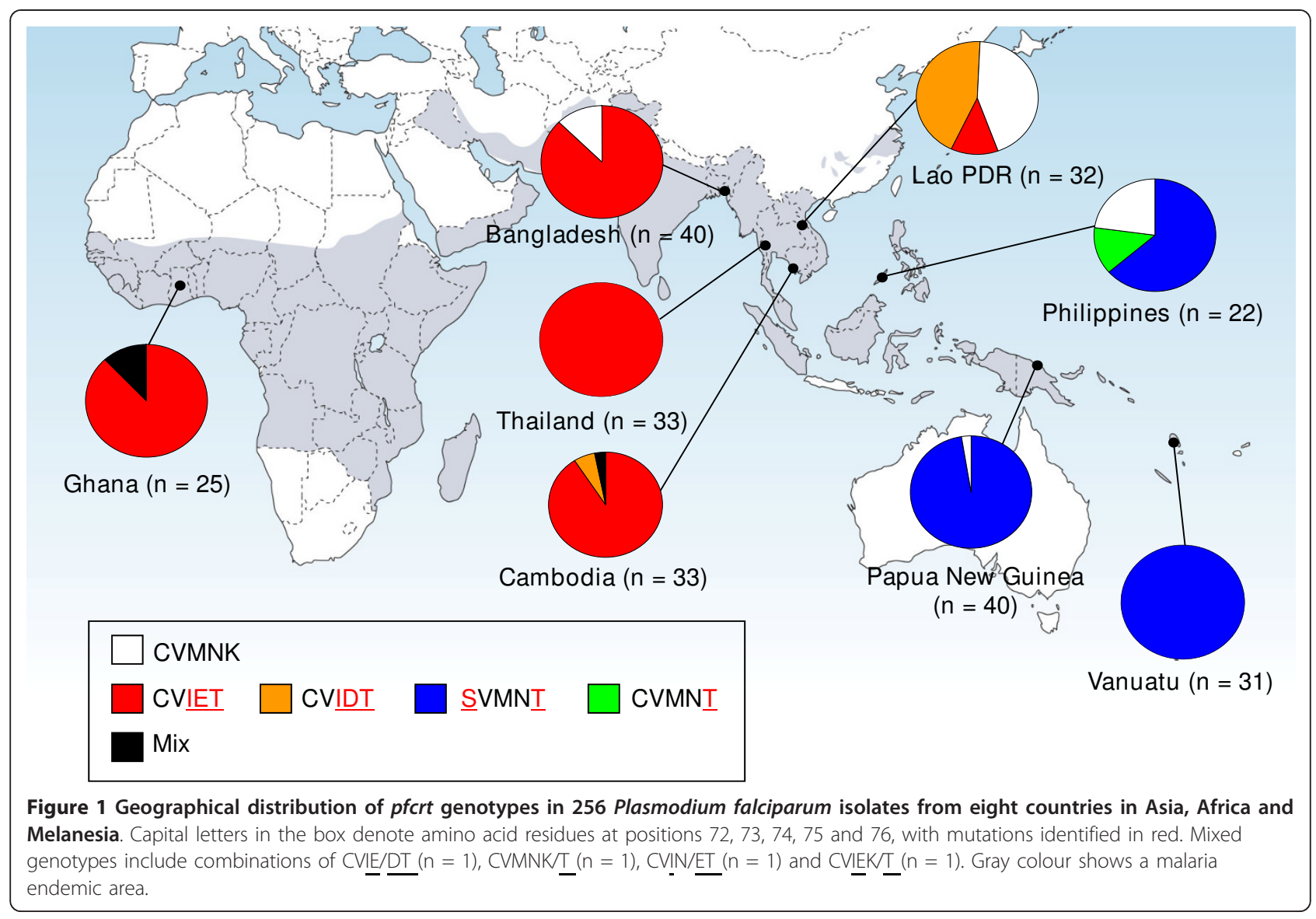




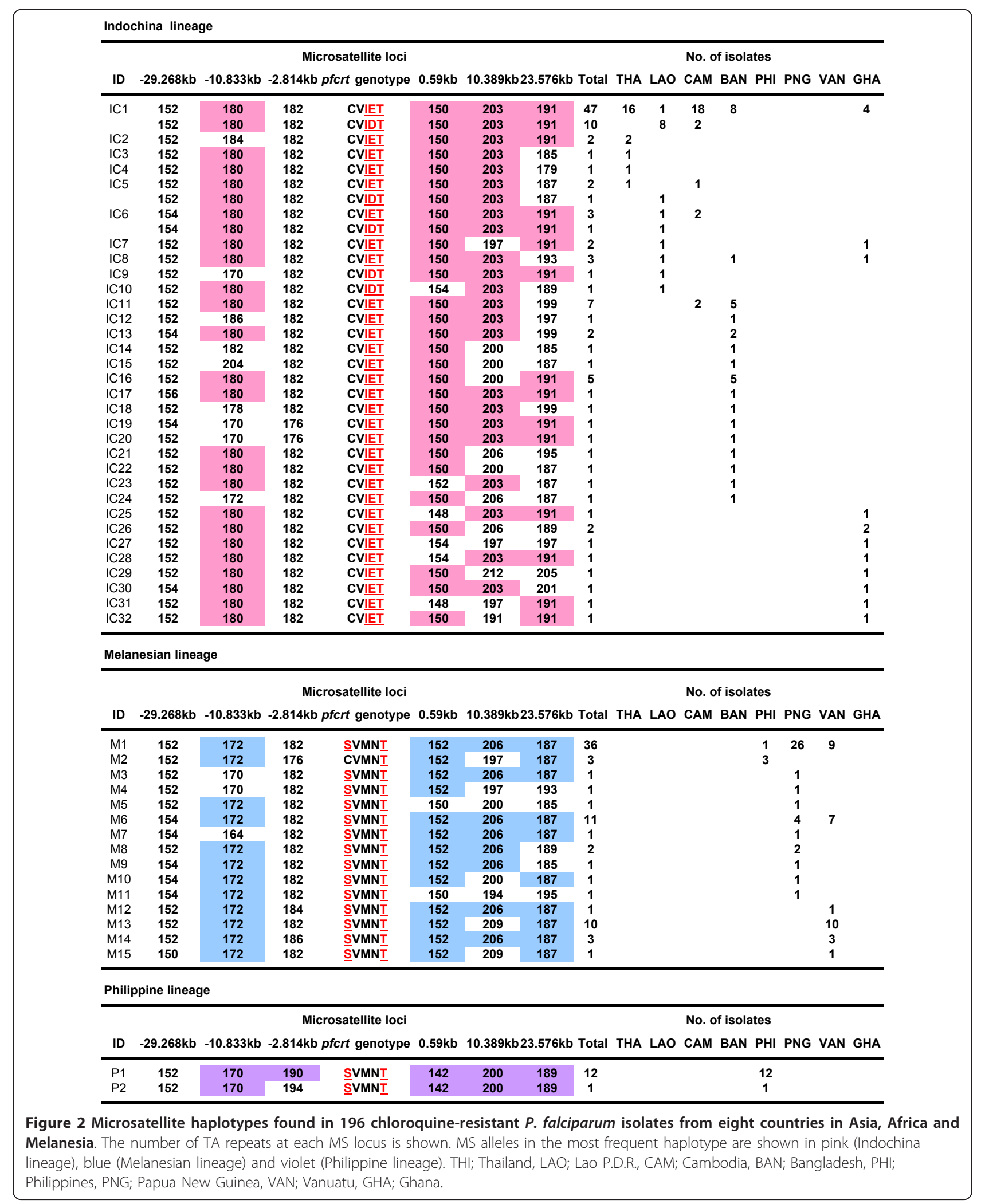




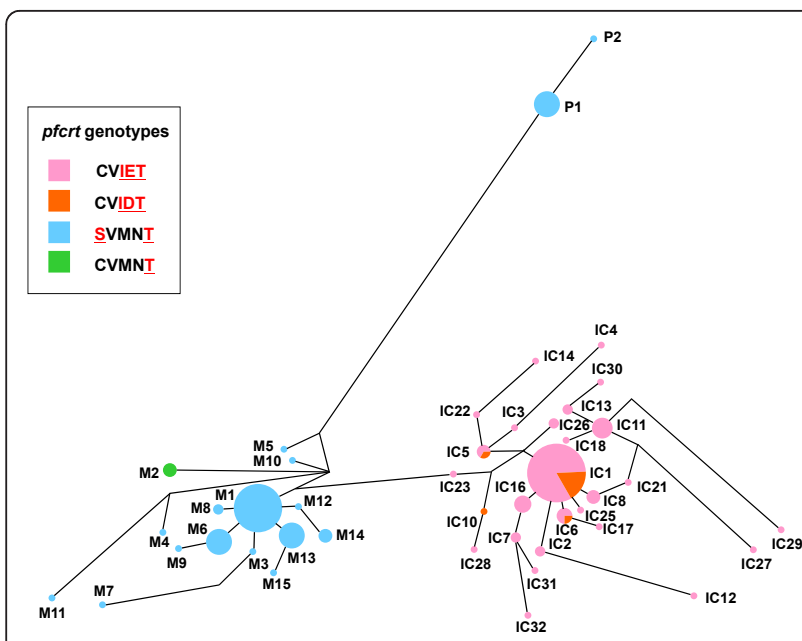

Figure 3 Haplotype network diagram in Plasmodium falciparum isolates harbouring pfcrt mutation. Network tree is shown according to pfcrt genotypes. The haplotype network was constructed for 196 P. falciparum isolates harbouring pfcrt mutation comprises 49 microsatellite haplotypes, based on allelic variations in six microsatellite loci flanking the pfcrt locus (see text for details). The size of each circle corresponds to the number of samples sharing the same haplotype, and the length of an edge is proportional to a variation in repeat number between two haplotypes. IC; Indochina lineage, M; Melanesian lineage, P; Philippine lineage.

observed in the Melanesian lineage [1], all isolates in the Philippine lineage harboured the SVMNT genotype.

The geographical distribution of the three major lineages described above was clearly distinctive (Figure $4)$. The Indochina lineage was widely distributed in Indochina and Africa, and this is consistent with previous reports [1,11]. Distribution of the Melanesian lineage was limited to Papua New Guinea and Vanuatu, except for four isolates (M1 and M2 found in the Philippines, which probably migrated from Melanesia).

\section{Discussion}

The present study using 256 P. falciparum isolates collected from Asia, Melanesia and Africa revealed only three major lineages of $C Q$ resistance originating in Indochina, Melanesia and the Philippines. No novel pfcrt genotype was identified in the present samples collected from large-scale survey. The number of CQmutant lineages observed in this study was identical to that in two previous studies that analysed 48 laboratoryadapted CQ-resistant parasite lines [1] and field isolates in the Philippines [14]. This observation supports the hypothesis that the emergence of CQ resistance is a rare event, but the reasons for this rare emergence of $C Q$ resistance remain to be clarified.

This study also revealed that a single CQ resistance lineage contained two mutant pfcrt genotypes; the

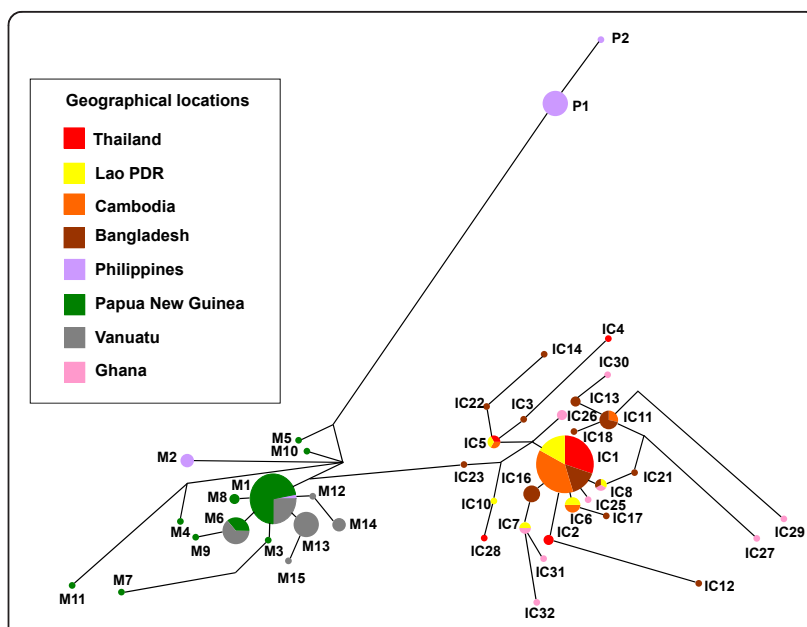

Figure 4 Haplotype network diagram in Plasmodium falciparum isolates harbouring pfcrt mutation. Network tree is shown according to countries where samples were taken. The haplotype network was constructed for 196 P. falciparum isolates harbouring pfcrt mutation comprises 49 microsatellite haplotypes, based on allelic variations in six microsatellite loci flanking the pfcrt locus (see text for details). The size of each circle corresponds to the number of samples sharing the same haplotype, and the length of an edge is proportional to a variation in repeat number between two haplotypes. IC; Indochina lineage, M; Melanesian lineage, P; Philippine lineage.

CVIET and CVIDT genotypes in the Indochina lineage, and the SVMNT and CVMNT genotypes in the Melanesian lineage. Similar findings have been reported previously; two mutant genotypes (CVIET and CVIES) in a single CQ-resistant lineage in Africa [11] and two mutant genotypes (CVMNT and CVMET) in a single CQ-resistant lineage in South America [1]. In the present study, CVIET was the predominant mutant genotype in Asia and Africa, except in Lao P.D.R., where the CVIDT genotype was more prevalent than CVIET. A high prevalence of the CVIET genotype supports the notion that this genotype is an ancestral mutant genotype in the Indochina lineage, from which the CVIDT genotype may have evolved. Similarly, the CVMNT genotype, which was observed in only one case, appears to be a descendent of the SVMNT genotype of the Melanesian lineage. These additional amino acid changes may have been generated after the emergence of CQ resistance, perhaps in the last 30-40 years [37]. Thus, it is likely that additional amino acid change(s) at positions 72-75 in pfcrt were recently generated in parasites harbouring the pfcrt K76T mutation.

The role of pfcrt mutations other than K76T remains to be fully elucidated. They may confer some benefit to CQ-resistant parasites, e.g., a small effect on CQ tolerance or compensation for impaired protein activity after the acquisition of the critical mutation K76T. For the conventional anti-malarial drug pyrimethamine, it is 
known that levels of resistance increase as mutations progressively accumulate in $d h f r$; relative $\mathrm{IC}_{50}$ values of pyrimethamine for mutant $d h f r$ genotypes, as compared to the wild $d h f r$ genotype, are 35 -fold higher in a single mutant and 1,111-fold higher in a quadruple mutant (highest resistance $d h f r$ genotype) [38]. Similarly, association between the accumulation of mutations in dhps and progressive increases of sulphadoxine resistance have also been reported [39]. Further studies are necessary to clarify the potential association between the accumulation of additional pfcrt mutations and augmentation of $\mathrm{CQ}$ resistance.

The present analysis did not identify a back mutation at position 76 in $p f c r t$, as observed in previous studies [26-28]. However, there remains the possibility that the back mutation has occurred in geographic areas not studied here. Most of the isolates sampled in the present study came from Asia and Melanesia, where CQ is still being used for treatment of Plasmodium vivax. Thus, the continuous CQ pressure present in Asia and Melanesia may be suppressing the expression of this back mutation. Further molecular epidemiological studies in different endemic areas having different histories of $C Q$ usage may be necessary in order to better understand the possible back mutation at the critical position 76 , which would indicate the recovery of CQ susceptibility.

The conclusions of the present study are limited to samples collected from the late 1990s to the mid 2000s in geographical areas where samples were collected. Although the intercontinental migration of $C Q$ resistance from Asia to Africa was already accomplished, the selection and spread of CQ resistance was not homogenously advanced in Asia. In fact, at the time of our sampling, while mutant $p f c r t$ alleles were almost fixed in Thailand and Cambodia, the selection seemed to be ongoing in Lao PDR, where nearly half of P. falciparum still harboured the wild $p f c r t$ genotype.

\section{Conclusions}

The present molecular analysis using samples across Asia, Africa and Melanesia provided two important insights into $C Q$ resistance in terms of malaria control. First, the emergence of novel CQ-mutant lineages among $P$. falciparum isolates is rare. Second, in isolates harbouring the K76T mutation, additional mutations in pfcrt other than K76T have readily been generated (in the last 30 years). These new mutations may confer stronger resistance to $\mathrm{CQ}$ than the K76T mutant. Further molecular monitoring of pfcrt genotypes should provide valuable information with regard to the current situation of CQ resistance and to the potential emergence of CQ-sensitive P. falciparum isolates in areas where $C Q$ use has been withdrawn.

\section{Additional material}

Additional file 1: PCR conditions for genotyping of pfcrt

Additional file 2: PCR conditions for determining variations of six microsatellite loci flanking pfcrt.

\section{Acknowledgements}

We would like to thank all participants in this study, and Hikota Osawa, Ilomo Hwaihwanje, and Aung Swi Prue Marma for their assistance with the field work. This study was supported by Grants-in-aid for Scientific Research from the Ministry of Education, Culture, Sports, Science and Technology of Japan [22406012, 23650211 and 23590498].

\section{Author details}

'Department of International Affairs and Tropical Medicine, Tokyo Women's Medical University School of Medicine, 8-1 Kawada-cho, Shinjuku-ku, Tokyo 162-8666, Japan. ²Department of Molecular Protozoology, Research Institute for Microbial Diseases, Osaka University, Suita, Osaka, Japan. ${ }^{3}$ Department of Biological, Environmental and Occupational Health Sciences, School of Public Health, University of Ghana, Legon, Ghana. ${ }^{4}$ National Center For Parasitology Entomology and Malaria Control, Phnom Pehn, Cambodia. ${ }^{5}$ Prevention Division, Hygiene Prevention Department, Ministry of Health, Vientiane, Lao P.D.R. ${ }^{6}$ Department of Entomology, Armed Forces Research Institute of Medical Sciences, Bangkok, Thailand. ${ }^{7}$ Department of Tropical Medicine and Parasitology, Dokkyo Medical University, Tochigi, Japan. ${ }^{8}$ Graduate School of International Health Development, Nagasaki University, Nagasaki, Japan. ${ }^{9}$ Department of Microbiology, Tumor and Cell Biology, Karolinska Institutet, Stockholm, Sweden. ${ }^{10}$ Department of Parasitology, Osaka City University Graduate School of Medicine, Osaka, Japan. ${ }^{11}$ Institute of Tropical Medicine, Nagasaki University, Nagasaki, Japan. ${ }^{12}$ Center for Health Research, Divine Word University, Madang, Papua New Guinea. ${ }^{13}$ Cell-free Science and Technology Research Center, and Venture Business Laboratory, Ehime University, Matsuyama, Ehime, Japan.

\section{Authors' contributions}

NT performed experiments, data analysis and paper writing. $T T 1, M D, L D, B K$, JS, MN, MS, JK, FH, TT2 and AK coordination of sampling. KT critically reviewed the manuscript. KT, HE and TM participated in acquisition of funding. TM made substantial contributions to study design, coordination of sampling, data analysis, paper writing and reviewing. All authors have read and approved the final manuscript.

\section{Competing interests}

The authors declare that they have no competing interests.

Received: 1 December 2011 Accepted: 28 March 2012 Published: 28 March 2012

\section{References}

1. Wootton JC, Feng X, Ferdig MT, Cooper RA, Mu J, Baruch DI, Magill AJ, Su XZ: Genetic diversity and chloroquine selective sweeps in Plasmodium falciparum. Nature 2002, 418:320-323.

2. Nair S, Williams JT, Brockman A, Paiphun L, Mayxay M, Newton PN, Guthmann JP, Smithuis FM, Hien TT, White NJ, Nosten F, Anderson TJ: A selective sweep driven by pyrimethamine treatment in Southeast Asian malaria parasites. Mol Biol Evol 2003, 20:1526-1536.

3. Roper C, Pearce R, Nair S, Sharp B, Nosten F, Anderson T: Intercontinental spread of pyrimethamine-resistant malaria. Science 2004, 305:1124.

4. Mita T, Tanabe K, Kita K: Spread and evolution of Plasmodium falciparm drug resistance. Parasitol Int 2009, 58:201-209.

5. Harinasuta $T$, Suntharasamai $P$, Viravan $C$ : Chloroquine-resistant falciparum malaria in Thailand. Lancet 1965, 2:657-660.

6. Moore DV, Lanier JE: Observations on two Plasmodium falciparum infections with an abnormal response to chloroquine. Am J Trop Med Hyg 1961, 10:5-9.

7. Meuwissen $\mathrm{JH}$ : The use of medicated salt in an antimalaria campaign in West New Guinea. Trop Geogr Med 1964, 16:245-255. 
8. Yung AP, Bennett NM: Chloroquine-resistant falciparum malaria in Papua New Guinea. Med J Australia 1976, 2:320-321.

9. Campbell CC, Chin W, Collins WE, Teutsch SM, Moss DM: Chloroquineresistant Plasmodium falciparu from East Africa: cultivation and drug sensitivity of the Tanzanian I/CDC strain from an American tourist. Lancet 1979, 2:1151-1154

10. Fogh S, Jepsen S, Effersoe P: Chloroquine-resistant Plasmodium falciparum malaria in Kenya. Trans R Soc Trop Med Hyg 1979, 73:228-229.

11. Ariey F, Fandeur T, Durand R, Randrianarivelojosia M, Jambou R, Legrand E, Ekala MT, Bouchier C, Cojean S, Duchemin JB, Robert V, Le Bras J, Mercereau-Puijalon O: Invasion of Africa by a single pfcrt allele of South East Asian type. Malar J 2006, 5:34

12. Fidock DA, Nomura T, Talley AK, Cooper RA, Dzekunov SM, Ferdig MT, Ursos LM, Sidhu AB, Naude B, Deitsch KW, Su XZ, Wootton JC, Roepe PD, Wellems TE: Mutations in the $P$. falciparum digestive vacuole transmembrane protein $P f C R T$ and evidence for their role in chloroquine resistance. Mol Cell 2000, 6:861-871.

13. Martin RE, Marchetti RV, Cowan Al, Howitt SM, Broer S, Kirk K: Chloroquine transport via the malaria parasite's chloroquine resistance transporter. Science 2009, 325:1680-1682.

14. Chen N, Kyle DE, Pasay C, Fowler EV, Baker J, Peters JM, Cheng Q: pfcrt Allelic types with two novel amino acid mutations in chloroquineresistant Plasmodium falciparum isolates from the Philippines. Antimicrob Agents Chemother 2003, 47:3500-3505

15. Best Plummer W, Pinto Pereira LM, Carrington CV: Pfcrt and pfmdr1 alleles associated with chloroquine resistance in Plasmodium falciparum from Guyana, South America. Mem Inst Oswaldo Cruz 2004, 99:389-392.

16. Restrepo-Pineda E, Arango E, Maestre A, Do Rosario VE, Cravo P: Studies on antimalarial drug susceptibility in Colombia, in relation to Pfmdr 1 and Pfcrt. Parasitology 2008, 135:547-553.

17. Nagesha HS, Casey GJ, Rieckmann KH, Fryauff DJ, Laksana BS, Reeder JC, Maguire JD, Baird JK: New haplotypes of the Plasmodium falciparum chloroquine resistance transporter (pfcrt) gene among chloroquineresistant parasite isolates. Am J Trop Med Hyg 2003, 68:398-402.

18. Hatabu T, Iwagami M, Kawazu S, Taguchi N, Escueta AD, Villacorte EA, Rivera PT, Kano S: Association of molecular markers in Plasmodium falciparum crt and $m d r 1$ with in vitro chloroquine resistance: a Philippine study. Parasitol Int 2009, 58:166-170.

19. Huaman MC, Yoshinaga K, Suryanatha A, Suarsana N, Kanbara H: Polymorphisms in the chloroquine resistance transporter gene in Plasmodium falciparum isolates from Lombok, Indonesia. Am J Trop Med Hyg 2004, 71:40-42

20. Lim P, Chy S, Ariey F, Incardona S, Chim P, Sem R, Denis MB, Hewitt S, Hoyer S, Socheat D, Mercereau-Puijalon O, Fandeur T: pfcrt polymorphism and chloroquine resistance in Plasmodium falciparum strains isolated in Cambodia. Antimicrob Agents Chemother 2003, 47:87-94.

21. Randrianarivelojosia M, Fidock DA, Belmonte O, Valderramos SG, MercereauPuijalon O, Ariey F: First evidence of pfcrt mutant Plasmodium falciparum in Madagascar. Trans R Soc Trop Med Hyg 2006, 100:826-830.

22. Awasthi G, Prasad GB, Das A: Population genetic analyses of Plasmodium falciparum chloroquine receptor transporter gene haplotypes reveal the evolutionary history of chloroquine-resistant malaria in India. Int J Parasitol 2011, 41:705-709.

23. Mita T, Kaneko A, Lum JK, Bwijo B, Takechi M, Zungu IL, Tsukahara T, Tanabe K, Kobayakawa T, Bjorkman A: Recovery of chloroquine sensitivity and low prevalence of the Plasmodium falciparum chloroquine resistance transporter gene mutation K76T following the discontinuance of chloroquine use in Malawi. Am J Trop Med Hyg 2003, 68:413-415.

24. Kublin JG, Cortese JF, Njunju EM, Mukadam RA, Wirima JJ, Kazembe PN, Djimde AA, Kouriba B, Taylor TE, Plowe CV: Reemergence of chloroquinesensitive Plasmodium falciparum malaria after cessation of chloroquine use in Malawi. J Infect Dis 2003, 187:1870-1875.

25. Laufer MK, Thesing PC, Eddington ND, Masonga R, Dzinjalamala FK, Takala SL, Taylor TE, Plowe CV: Return of chloroquine antimalarial efficacy in Malawi. N Engl J Med 2006, 355:1959-1966.

26. Chen N, Gao Q, Wang S, Wang G, Gatton M, Cheng Q: No Genetic bottleneck in Plasmodium falciparum wild-type pfcrt alleles reemerging in Hainan Island, China, following high-level chloroquine resistance. Antimicrob Agents Chemother 2008, 52:345-347.

27. Mita T, Kaneko A, Lum JK, Zungu IL, Tsukahara T, Eto H, Kobayakawa T, Bjorkman A, Tanabe K: Expansion of wild type allele rather than back mutation in pfcrt explains the recent recovery of chloroquine sensitivity of Plasmodium falciparum in Malawi. Mol Biochem Parasitol 2004, 135:159-163.

28. Laufer MK, Takala-Harrison S, Dzinjalamala FK, Stine OC, Taylor TE, Plowe CV: Return of chloroquine-susceptible falciparum malaria in Malawi was a reexpansion of diverse susceptible parasites. J Infect Dis 2010, 202:801-808.

29. Dysoley L, Kaneko A, Eto H, Mita T, Socheat D, Borkman A, Kobayakawa T: Changing patterns of forest malaria among the mobile adult male population in Chumkiri District, Cambodia. Acta Trop 2008, 106:207-212.

30. Marma AS, Mita T, Eto H, Tsukahara T, Sarker S, Endo H: High prevalence of sulfadoxine/pyrimethamine resistance alleles in Plasmodium falciparum parasites from Bangladesh. Parasitol Int 2010, 59:178-182.

31. Sakihama N, Nakamura M, Palanca AA Jr, Argubano RA, Realon EP, Larracas AL, Espina RL, Tanabe K: Allelic diversity in the merozoite surface protein 1 gene of Plasmodium falciparum on Palawan Island, the Philippines. Parasitol Int 2007, 56:185-194.

32. Mita T, Kaneko A, Hombhanje F. Hwaihwanje I, Takahashi N, Osawa H, Tsukahara T, Masta A, Lum JK, Kobayakawa T, Ishizaki T, Björkman A: Role of pfmdr1 mutations on chloroquine resistance in Plasmodium falciparum isolates with pfcrt K76T from Papua New Guinea. Acta Trop 2006, 98:137-144.

33. Sakihama N, Kaneko A, Hattori T, Tanabe K: Limited recombination events in merozoite surface protein-1 alleles of Plasmodium falciparum on islands. Gene 2001, 279:41-48

34. Nash D, Nair S, Mayxay M, Newton PN, Guthmann JP, Nosten F, Anderson TJ: Selection strength and hitchhiking around two anti-malarial resistance genes. Proc Biol Sci 2005, 272:1153-1161.

35. Bandelt $H J$, Forster $P$, Rohl A: Median-joining networks for inferring intraspecific phylogenies. Mol Biol Evol 1999, 16:37-48.

36. Mehlotra RK, Mattera G, Bockarie MJ, Maguire JD, Baird JK, Sharma YD, Alifrangis M, Dorsey G, Rosenthal PJ, Fryauff DJ, Kazura JW, Stoneking M Zimmerman PA: Discordant patterns of genetic variation at two chloroquine resistance loci in worldwide populations of the malaria parasite Plasmodium falciparu. Antimicrob Agents Chemother 2008, 52:2212-2222.

37. Wellems TE, Plowe CV: Chloroquine-resistant malaria. J Infect Dis 2001, 184:770-776

38. Lozovsky ER, Chookajorn T, Brown KM, Imwong M, Shaw PJ, Kamchonwongpaisan S, Neafsey DE, Weinreich DM, Hartl DL: Stepwise acquisition of pyrimethamine resistance in the malaria parasite. Proc Nat Acad Sci USA 2009, 106:12025-12030.

39. Triglia T, Wang P, Sims PF, Hyde JE, Cowman AF: Allelic exchange at the endogenous genomic locus in Plasmodium falciparu proves the role of dihydropteroate synthase in sulfadoxine-resistant malaria. EMBO J 1998, 17:3807-3815.

doi:10.1186/1475-2875-11-92

Cite this article as: Takahashi et al:: Large-scale survey for novel genotypes of Plasmodium falciparum chloroquine-resistance gene pfcrt. Malaria Journal 2012 11:92

\section{Submit your next manuscript to BioMed Central and take full advantage of:}

- Convenient online submission

- Thorough peer review

- No space constraints or color figure charges

- Immediate publication on acceptance

- Inclusion in PubMed, CAS, Scopus and Google Scholar

- Research which is freely available for redistribution 